\title{
Endovascular repair of de novo post- stenotic aortic coarctation aneurysms with complex collateral supply: two cases with long and medium term follow-up
}

\author{
Omar Abdel-Hadi ${ }^{1}$, John Thomson ${ }^{2,3}$ and Simon J. McPherson ${ }^{1,3^{*}}$ (i)
}

\begin{abstract}
Purpose: To report the technical details and outcomes of the endovascular repair of two cases of de novo poststenotic aortic coarctation aneurysms complicated by complex collateral supply.

Case presentations: Two patients with thoracic aortic aneurysms complicated by complex aneurysm sac collaterals distal to a previously untreated thoracic aortic coarctation have been treated at our institution. Open surgical intervention was deemed to carry a high risk of haemorrhage due to the degree and complexity of arterial collateralisation. In the first case, selective embolisation of collateral vasculature was performed prior to successful exclusion of the aneurysm with a thoracic endovascular stent-graft and then balloon-expandable stent dilatation of the coarctation stenosis. In the second case, the additional technique of using a jailed sheath within the aneurysm sac allowed for selective embolisation of previously inconspicuous collaterals after deployment of the stent-graft and stent combination.

Results: Technical success was achieved in both patients with successful occlusion of the aneurysm, with no recorded complications or aneurysm sac perfusion in the long and medium term follow up periods respectively.

Conclusion: De novo post stenotic aortic coarctation aneurysms are rare. Endovascular repair is a safe and durable technique that provides a less invasive alternative to open surgical repair. The use of a jailed sheath allows for complete selective embolisation of complex collaterals avoiding a type II aneurysm endoleak.
\end{abstract}

Keywords: Percutaneous, Thoracic coarctation aneurysm, Thoracic aortic stent graft/repair, TEVAR, Aortic coarctation stent, Embolisation

\section{Background}

Thoracic aortic coarctation (TAC) is a focal aortic constriction typically at the ductus or ligamentum arteriosum. It accounts for 5-8\% of congenital cardiac defects (Tynan et al. 1990; Warnes et al. 2008). Consequent increased cardiac afterload results in hypertension, left ventricular hypertrophy, and congestive cardiac failure

\footnotetext{
* Correspondence: simon.mcpherson@nhs.net

'Department of Radiology, Leeds Teaching Hospitals NHS Trust, Leeds General Infirmary, Great George Street, Leeds, West Yorkshire LS1 3EX, UK

${ }^{3}$ University of Leeds, Leeds, UK

Full list of author information is available at the end of the article
}

(Warnes et al. 2008). Most present in childhood when open surgical repair is often performed. Surgical approaches include excision with end-to-end anastomosis, extended end to side anastomosis (particularly in infants), synthetic patch aortoplasty, subclavian artery flap aortoplasty and interposition tube grafts (Khavandi et al. 2013). Presentation as an adult occurs in $15-20 \%$ who are asymptomatic in childhood. The natural history of untreated adult TAC is a mortality rate approaching $75 \%$ by the fifth decade (Campbell 1970; Erbel et al. 2014)., The preferred treatment in older children and adult patients is endovascular due to its lower morbidity

\section{Springer Open}

() The Author(s). 2021 Open Access This article is licensed under a Creative Commons Attribution 4.0 International License, which permits use, sharing, adaptation, distribution and reproduction in any medium or format, as long as you give appropriate credit to the original author(s) and the source, provide a link to the Creative Commons licence, and indicate if changes were made. The images or other third party material in this article are included in the article's Creative Commons licence, unless indicated otherwise in a credit line to the material. If material is not included in the article's Creative Commons licence and your intended use is not permitted by statutory regulation or exceeds the permitted use, you will need to obtain permission directly from the copyright holder. To view a copy of this licence, visit http://creativecommons.org/licenses/by/4.0/. 
and mortality compared with surgery (Forbes and Gowda 2014).

Post-surgical aneurysms in TAC are well recognised but aneurysms are much rarer in untreated cases (Khavandi et al. 2013; Turner and Gaines 2007). Thoracic Endovascular Aneurysm Repair (TEVAR) is the accepted first-line treatment for post-surgical aneurysms, given the mortality rate of $14 \%$ for repeat open surgery (Ince et al. 2003). Successful open surgery for de-novo TAC associated aneurysms is limited to case reports (Ruys et al. 2014; Ananiadou et al. 2012; Careaga-Reyna et al. 2009). Stent-grafts in combination with other endovascular techniques offer a flexible alternative to cope with anatomic complexity (Kutty et al. 2008).

We report two patients with de novo aneurysms distal to a TAC, with associated extensive aneurysm collateralisation, treated by complex endovascular repair.

\section{Case presentation}

\section{Case 1}

A 28-year-old male presented in 2010 with chest pain following amphetamine use. CXR revealed a grossly enlarged and peripherally calcified descending thoracic aortic aneurysm with rib notching (Fig. 1). CT angiogram confirmed a $5.7 \mathrm{~cm}$ calcified aneurysm distal to a $5 \mathrm{~mm}$ lumen TAC (Fig. 2), and florid predominantly posterior collaterals. The chest pain persisted despite medical therapy. Alternative causes for the pain were

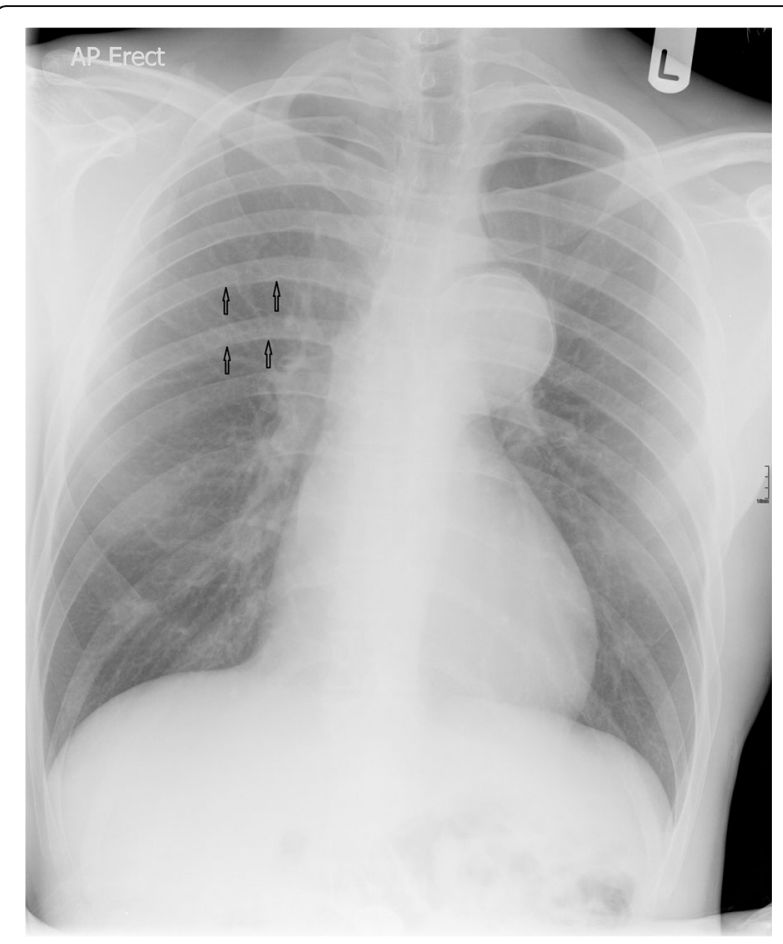

Fig. 1 Presenting chest radiograph with a large calcified aortic aneurysm and rib notching in the right upper zone (Black Arrows) excluded. Discussion at the complex cardiothoracic disease multi-disciplinary team (MDT) meeting concluded that the extent of collateralisation posed a high risk of uncontrollable bleeding. An endovascular approach was preferred, this was agreed with the patient.

Under general anaesthesia (GA), with a cardiothoracic team on stand-by, an 18Fr sheath (Cook, Bloomington, IN) was inserted via a right common femoral artery (CFA) surgical cut down with a left CFA 6Fr sheath. The procedure predates our routine use of large arterial access closure devices. The small TAC lumen was hard to identify within the large aneurysm. It was eventually crossed with a 4Fr vertebral catheter and hydrophilic wire (Terumo, Japan). A $260 \mathrm{~cm}$ Amplatz wire (Cook) was placed across the TAC into the left subclavian artery (LSCA) for emergency TEVAR or balloon control in the event of bleeding.

Over 10 intercostal and bronchial arteries arising from the aneurysm sac were coiled using a combination of 0.035 in. Nester (Cook), 0.035 MReye coils (Cook) Trufill platinum micro coils (Cordis, Miami, Florida) and one Amplatz AVP1 $10 \mathrm{~mm}$ plug (AGA Medical, Plymouth, Minnesota,). TAC $8 \mathrm{~mm}$ pre-dilatation facilitated TEVA R delivery. A $21 \mathrm{~mm} \times 100 \mathrm{~mm}$ Gore TAG device (W. L. Gore, Flagstaff, AZ) was deployed immediately distal to the LSCA. A $39 \mathrm{~mm}$ length uncovered CP stent (NuMED, Hopkinton, New York,) mounted onto a 16 $\mathrm{mm}$ outer BIB balloon (NuMED) was delivered flush with the cranial stent graft with serial inflations leading to good apposition resulting in almost complete abolition of the coarctation and exclusion of the aneurysm (Fig. 3). Procedure time was $5 \mathrm{~h}$. CT angiography at $72 \mathrm{~h}$ confirmed complete aneurysm exclusion.

Over 7 years CT surveillance showed aneurysm sac shrinkage to $4.2 \mathrm{~cm}$ with no endoleak. Eight years post operatively the patient presented with left sided weakness secondary to emboli from culture negative infection associated with intravenous drug use. Echocardiography identified a mitral valve vegetation. CT angiogram and PET-CT showed no evidence of infection at the site of the coarctation and aneurysm repair. Treatment was with long term IV antibiotics. At 10 years CT follow-up the aneurysm remains excluded and at a stable reduced size with no recurrence of the TAC.

\section{Patient 2}

A 29 year old female was referred from another regional specialist cardiothoracic centre. She was well until 2017 when she developed a painful left foot. Septic emboli due a Streptococcal sanguis endarteritis at the site of previously undiagnosed coarctation, with a $3.5 \mathrm{~cm}$ aneurysm distal to it, was diagnosed. The aneurysm had numerous large collateral vessels, and two lateral out-pouchings measuring $6 \times 19 \mathrm{~mm}$ and 


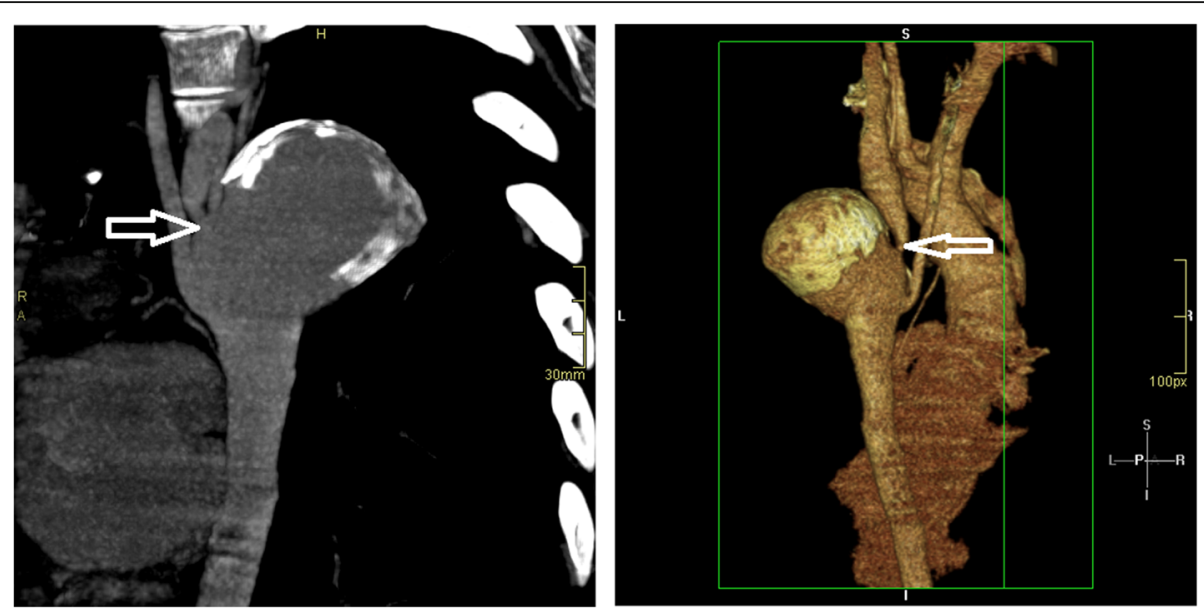

Fig. 2 Cross sectional reformats demonstrating aneurysm formation in an aortic coarctation (White arrows)

$11 \times 9 \mathrm{~mm}$ which were considered most likely to be pseudoaneurysms (Fig. 4). After 6 months of intravenous antibiotic therapy and imaging surveillance, she was referred for operative treatment. CT angiogram demonstrated an $8 \mathrm{~mm}$ coarctation $3 \mathrm{~cm}$ distal to the LSCA with an unchanged aneurysm (Fig. 4). Considering the infectious clinical presentation, preference to avoid an interposition graft with a suture line and high bleeding risk the MDT offered an endovascular repair.

Under GA, aneurysm sac collaterals were embolised via a 5Fr right CFA sheath using Trufill micro-coils (Cordis) and Amplatzer AVP4 vascular plugs (AGA Medical). These included superior intercostal arteries, bronchial arteries, broncho-intercostal arteries, and posterior intercostals connecting to the internal mammary arteries. No residual aneurysm sac collaterals were seen on completion aortic angiography. Surgical formation of a $10 \mathrm{~mm}$ distal aortic conduit was required for TEVAR delivery as the $5 \mathrm{~mm}$ iliac arteries were too narrow in calibre. The tip of an $80 \mathrm{~cm}$ 5Fr Flexor sheath (Cook) from a left CFA access was left within the aneurysm sac in case collateral embolisation was incomplete. A $31 \mathrm{~mm} \times 100 \mathrm{~mm}$ Gore TAG stent graft (W. L. Gore) was deployed distal to the origin of the LSCA. A $39 \mathrm{~mm}$ length covered CP stent (NuMED) was dilated to $24 \mathrm{~mm}$ to treat the aortic coarctation (Fig. 5).
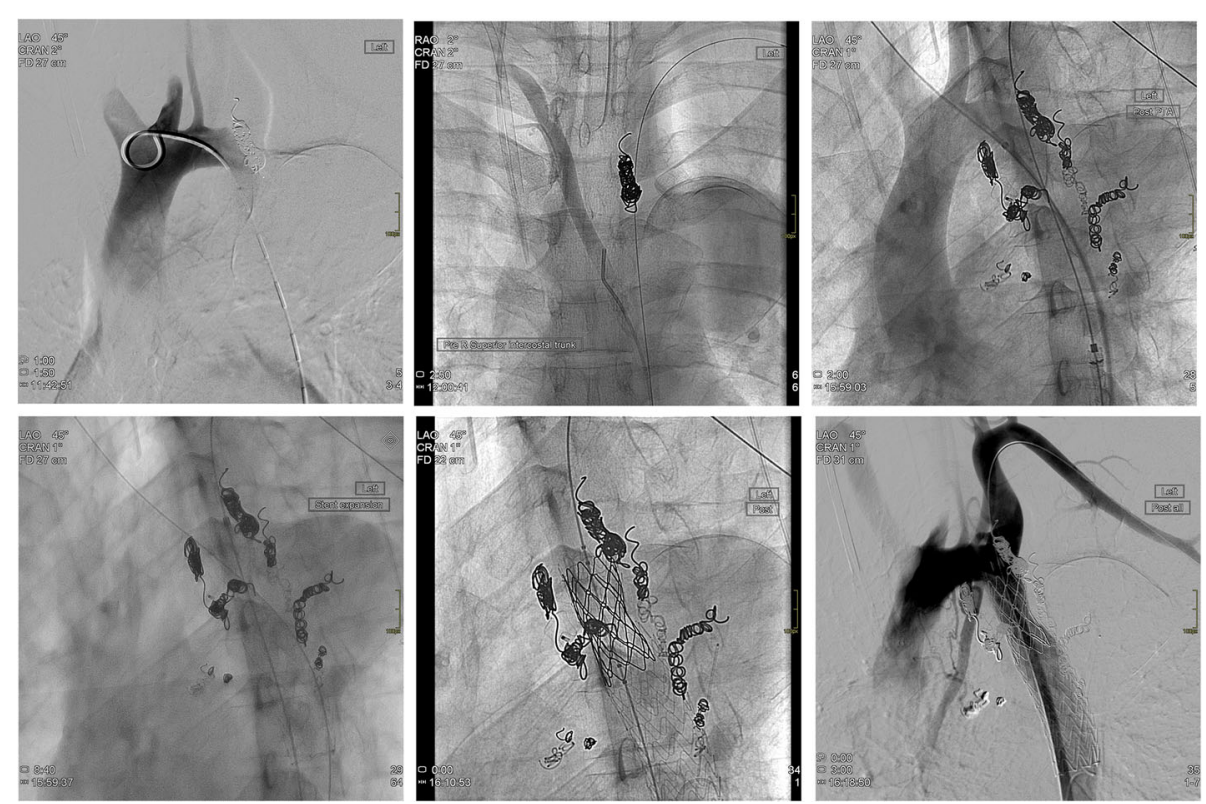

Fig. 3 Sequential embolization of collaterals followed by insertion of self-expanding and then balloon mounted stent grafts. Successful embolization and stent-grafting with no endoleak 

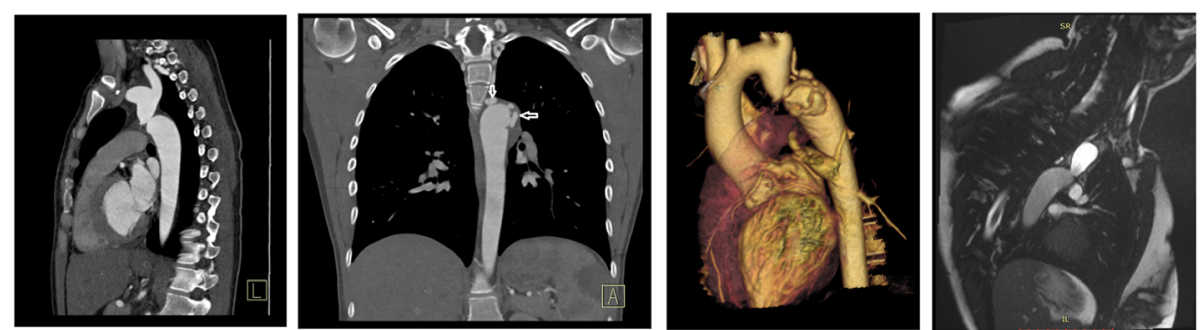

Fig. 4 Pre operative imaging demonstrating a significant post ductal aortic coarctation. Multiple aneurysms surrounding the coarctation (white arrows)

After exclusion of the aneurysm, angiography via the long sheath in the aneurysm sac demonstrated two patent non-embolised collateral branches (Fig. 5). These were embolised using a catheter and Progreat microcatheter (Terumo, Japan) combination and $10 \times 14$ micronester coils (Cook). No complication occurred. A 5 day post-operative CT showed no endoleak. She remains asymptomatic over 3 years of follow-up with a stable aneurysm sac and no endoleak.

\section{Discussion}

The two cases were performed 7 years apart reflecting the rarity of de novo TAC aneurysms with complex aneurysm sac collaterals. Endovascular intervention was a safe and durable treatment.

A balloon expandable stent graft was used in the second case as the seal zone was shorter than in the first case and if slippage of the TAG stent graft had occurred during the balloon expandable stent-graft deployment the seal zone would have been maintained.

In case one, successful embolisation of all complex collateral arteries was achieved prior to aneurysm and TAC treatment. In case two, persistent collaterals were present despite apparent complete embolisation before the aneurysm and TAC treatment. The aneurysm sac "jailed" sheath ensured two further branches were embolised post stent-grafting. Without a jailed sheath embolisation of the persisting collaterals would not have been possible with a high risk of type 2 endoleak. The jailed sheath allowed alternative embolisation techniques, such as cohesive liquid embolic agents (e.g.Onyx (ev3, Irvine, $\mathrm{CA})$, glue, gelatin sponge or thrombin, if the persisting collaterals could not be catheterised,.

\section{Conclusion}

Endovascular repair of de novo post stenotic aortic coarctation is a safe and durable technique that provides a

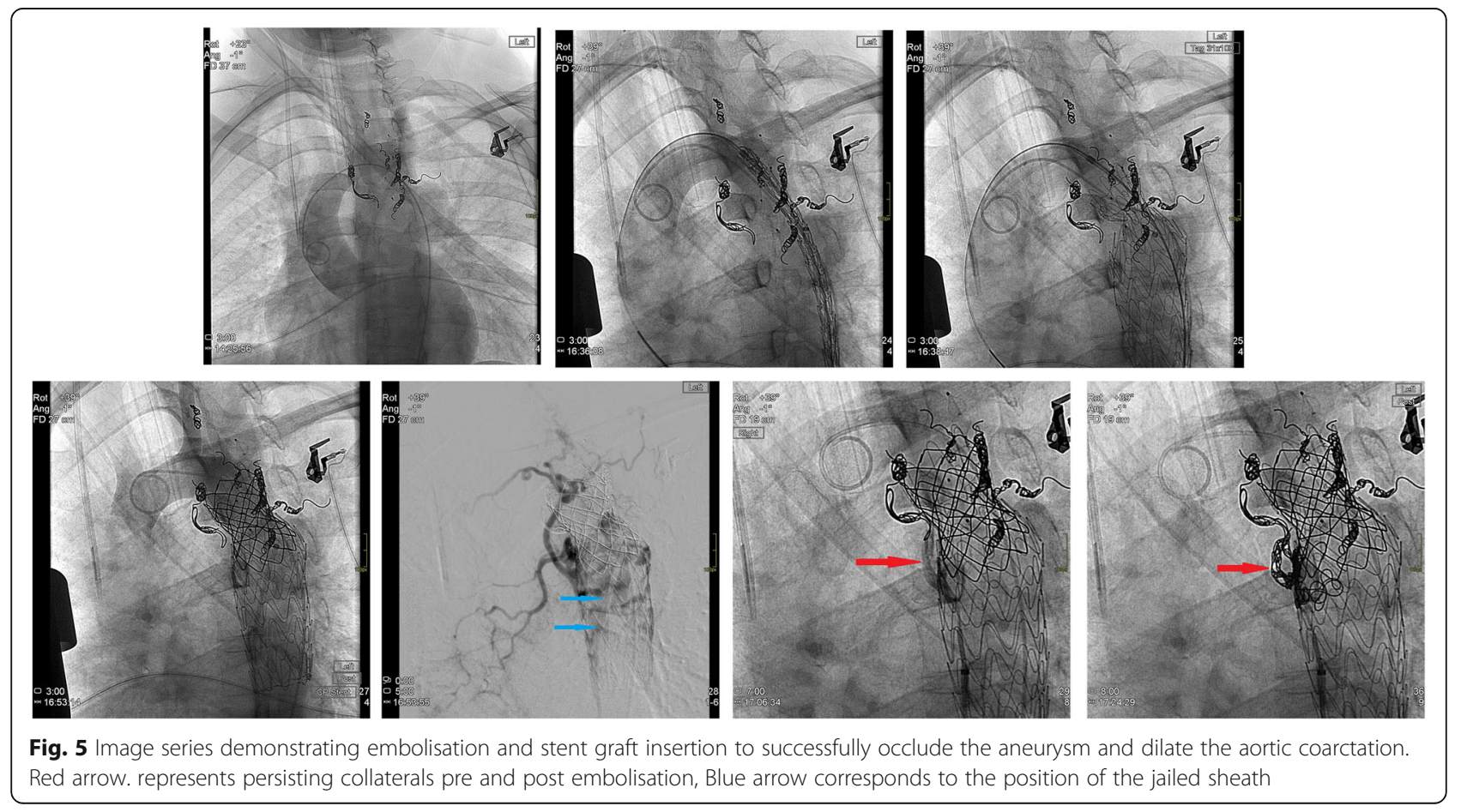


less invasive alternative to open surgical repair. An endovascular approach allows exclusion of complex collateralised aneurysms without the additional haemorrhage risk of open surgical repair. The jailed sheath technique allows for complete selective embolisation to ensure technical success without a type II endoleak.

\section{Acknowledgements}

Not applicable.

\section{Authors' contributions}

Dr. McPherson and Dr. Thomson performed the procedures. Dr. Abdel-Hadi wrote the manuscript with Dr. McPherson's support. Dr. McPherson and Dr. Thomson reviewed the case report manuscript. All authors read and approved the final report manuscript.

\section{Funding}

This study was not supported by any funding.

\section{Availability of data and materials}

Not Applicable.

\section{Ethics approval and consent to participate}

All procedures performed in studies involving human participants were in accordance with the ethical standards of the institutional and/or national research committee and with the 1964 Helsinki declaration and its later amendments or comparable ethical standards.

\section{Consent for publication}

Written informed consent was obtained from the patients for publication of this case report and any accompanying images.

\section{Competing interests}

The authors declare that they have no conflict of interest.

\section{Author details}

'Department of Radiology, Leeds Teaching Hospitals NHS Trust, Leeds General Infirmary, Great George Street, Leeds, West Yorkshire LS1 3EX, UK. ${ }^{2}$ Department of Adult Congenital Heart Disease, Leeds Teaching Hospitals NHS Trust, Leeds General Infirmary, Leeds LS1 3EX, West Yorkshire. LS1 3EX, UK. ${ }^{3}$ University of Leeds, Leeds, UK.

Received: 4 November 2020 Accepted: 7 December 2020

Published online: 11 January 2021

\section{References}

Campbell M (1970) Natural history of coarctation of the aorta. Br Heart J 32(5): 633-640

Careaga-Reyna G, Ramirez-Vargas AF, Hernandez-Magro RM, Arguero-Sanchez R (2009) Thoracic aortic aneurysm associated with aortic coarctation without previous surgery: report of two cases. Cir Cir 77(1):61-63

Erbel R, Aboyans V, Boileau C, Bossone E, Di Bartolomeo R, Eggebrecht H et al (2014) 2014 ESC Guidelines on the diagnosis and treatment of aortic diseases: Document covering acute and chronic aortic diseases of the thoracic and abdominal aorta of the adult. The Task Force for the Diagnosis and Treatment of Aortic Diseases of the European Society of Cardiology (ESC). Eur Heart J 35(41):2873-2926 [cited 2020 Feb 25]. Available from: http://www.ncbi.nlm.nih.gov/pubmed/25173340

Forbes TJ, Gowda ST (2014) Intravascular stent therapy for coarctation of the aorta. Methodist Debakey Cardiovasc J 10(2):82-87

Ince H, Petzsch M, Rehders T, Kische S, Korber T, Weber F et al (2003) Percutaneous endovascular repair of aneurysm after previous coarctation surgery. Circulation 108(24):2967-2970

Khavandi A, Bentham J, Marlais M, Martin RP, Morgan GJ, Parry AJ, et al. Transcatheter and endovascular stent graft management of coarctationrelated pseudoaneurysms. Heart. 2013 99(17):1275-1281. [cited 2020 Feb 22]; Available from: http://www.ncbi.nlm.nih.gov/pubmed/23825097

Kutty S, Greenberg RK, Fletcher S, Svensson LG, Latson LA (2008) Endovascular stent grafts for large thoracic aneurysms after coarctation repair. Ann Thorac Surg 85(4):1332-1338
Ananiadou OG, Koutsogiannidis C, Ampatzidou F, Drossos GE (2012) Aortic Root Aneurysm in an Adult Patient With Aortic Coarctation: A Single-Stage Approach. Interact Cardiovasc Thorac Surg 15(3)

Ruys TPE, Bekkers JA, Duvekot JJ, Roos-Hesselink JW (2014) A pregnant patient with native aortic Coarctation and aneurysm. AORTA 2(3):110-112

Turner DR, Gaines PA (2007) Endovascular management of coarctation of the aorta. Semin Interv Radiol 24:153-166 [cited 2020 Feb 22]Available from: http://www.ncbi.nlm.nih.gov/pubmed/21326793

Tynan M, Finley JP, Fontes V, Hess J, Kan J (1990) Balloon angioplasty for the treatment of native coarctation: results of Valvuloplasty and angioplasty of congenital anomalies registry. Am J Cardiol 65(11):790-792

Warnes CA, Williams RG, Bashore TM, Child JS, Connolly HM, Dearani JA et al (2008) ACC/AHA 2008 guidelines for the Management of Adults with congenital heart disease: executive summary: a report of the American College of Cardiology/American Heart Association task force on practice guidelines writing committee to develop guidelines for. Circulation 118(23): 2395-2451

\section{Publisher's Note}

Springer Nature remains neutral with regard to jurisdictional claims in published maps and institutional affiliations.

\section{Submit your manuscript to a SpringerOpen ${ }^{\circ}$ journal and benefit from:}

- Convenient online submission

- Rigorous peer review

- Open access: articles freely available online

High visibility within the field

- Retaining the copyright to your article

Submit your next manuscript at $\boldsymbol{\nabla}$ springeropen.com 